TRANSACTIONS OF THE

AMERICAN MATHEMATICAL SOCIETY

Volume 348, Number 1, January 1996

\title{
THE SPACES OF INDEX ONE MINIMAL SURFACES AND STABLE CONSTANT MEAN CURVATURE SURFACES EMBEDDED IN FLAT THREE MANIFOLDS
}

\author{
MANUEL RITORÉ AND ANTONIO ROS
}

\begin{abstract}
It is proved that the spaces of index one minimal surfaces and stable constant mean curvature surfaces with genus greater than one in (non fixed) flat three manifolds are compact in a strong sense: given a sequence of any of the above surfaces we can extract a convergent subsequence of both the surfaces and the ambient manifolds in the $C^{k}$ topology. These limits preserve the topological type of the surfaces and the affine diffeomorphism class of the ambient manifolds.

Some applications to the isoperimetric problem are given.
\end{abstract}

\section{INTRODUCTION}

In this paper we study minimal and constant mean curvature surfaces as critical points of variational problems. The former have critical area for all variations with compact support, the latter only for those preserving the volume "enclosed" by the surface. These surfaces sometimes come up associated to naturally occurring phenomena, and in this case they additionally exhibit some kind of stability by means of the second variation of the area.

If $M$ is an orientable minimal surface immersed in an orientable flat three dimensional manifold $N$, the second variation formula of the area is the quadratic form, called the index form of $M$, associated to the operator $L=\Delta+|\sigma|^{2}$, where $\Delta$ is the Laplacian on $M$ and $|\sigma|^{2}$ is the square of the norm of the second fundamental form $\sigma$ of the immersion. For any relatively compact domain $\Omega \subset M$, we define the index of $\Omega$, Ind $(\Omega)$, to be the number of negative eigenvalues for the Dirichlet problem of $L$ on $\Omega$. The index of $M$, $\operatorname{Ind}(M)$, is the supremum of all numbers $\operatorname{Ind}(\Omega)$ when $\Omega$ varies on $M$. Index zero minimal surfaces are those that minimize area on each relatively compact domain up to second order (these surfaces are usually called stable, but we reserve this name for the constant mean curvature case, see below). If the surfaces are complete and orientable and the ambient space is orientable with non negative scalar curvature, they have been studied by Fischer-Colbrie and Schoen [FCS], see the paper by do Carmo and Peng [dCP] for the case $N=\mathbb{R}^{3}$. It follows from their results that if the ambient manifold $N$ is flat then index zero minimal surfaces are totally geodesic. Index one minimal surfaces arise naturally

Received by the editors November 18, 1994 and, in revised form, March 27, 1995.

1991 Mathematics Subject Classification. Primary 53A10; Secondary 49Q20.

Key words and phrases. Minimal surfaces, constant mean curvature surfaces, index one, stability, isoperimetric problem.

Both authors partially supported by DGICYT grant PB91-0731. 
in minimax methods. A large number of such surfaces have been constructed by Pitts $[\mathrm{P}]$, Pitts and Rubinstein [PR], Hass, Pitts and Rubinstein [HPR] and Meeks $[\mathrm{M}]$. Orientable index one minimal surfaces in $\mathbb{R}^{3}$ have been studied by López and Ros in $[\mathrm{LR}]$ and by Montiel and Ros, see [MoR].

A constant mean curvature surface is called volume preserving stable if the second derivative of the area is non negative for compactly supported variations that preserve the signed volume enclosed by the surface, see [BdCE]. We shall refer to these surfaces simply as stable surfaces. If $M$ is an orientable surface immersed with constant mean curvature in an orientable flat three manifold, then $M$ is stable if, for any smooth function $u$ with compact support and such that $\int_{M} u d A=0$, we have:

$$
I(u)=\int_{M}\left\{|\nabla u|^{2}-|\sigma|^{2} u^{2}\right\} d A \geq 0 .
$$

As in the minimal case, $I$ is called the index form of $M$, and is the quadratic form associated to the operator $\Delta+|\sigma|^{2}$. Note that stability implies that the index of $M$ is less than or equal to one.

It is known that complete orientable non compact stable surfaces in flat three manifolds are totally geodesic, see [S], and that compact orientable stable surfaces have genus less than or equal to five, see $[\mathrm{Y}]$ or $[\mathrm{RR}]$. When the genus of the surface is zero then it must be an umbilical sphere, which is always stable. If the genus is one, it has been obtained by the authors in $[R R]$ that the surface must be a flat torus. For higher genus, many examples have been found by Ross [Ro], who has proved that Schwarz's $\mathcal{P}$ minimal surface of genus three is stable in the cubic three torus. He has also pointed that minimal surfaces close enough to Schwarz's surface in three tori close to the cubic three torus are also stable.

We prove in this paper compactness results for the space of compact orientable index one minimal surfaces in flat three tori and for the space of compact stable surfaces with genus greater than or equal to two embedded in non fixed complete orientable flat three manifolds. More precisely, for index one minimal surfaces we obtain in Theorem 3.2 that

From every sequence of compact orientable index one minimal surfaces embedded in flat three tori we can extract, up to scaling, a convergent subsequence from both the tori and the surfaces to an index one minimal surface embedded in the limit three torus.

The above surfaces converge in the $C^{k}$ topology, $k \geq 2$, and their topology is preserved in the limit. In particular, we obtain that the space of flat three tori, modulo scaling, that admit orientable embedded index one minimal surfaces is compact. So, most of these tori do not admit this kind of surfaces. Note that they all admit non-orientable compact minimal surfaces with index less than or equal to one, see $[\mathrm{HPR}]$ and $[\mathrm{M}]$. Another consequence is the finiteness of the number of homotopy classes of orientable index one embedded minimal surfaces in flat three tori.

For stable constant mean curvature surfaces we have, see Theorem 4.2, that

Let $M_{n} \subset N_{n}, n \in \mathbb{N}$, be a sequence of compact orientable stable surfaces embedded in complete orientable flat 3-manifolds. Assume that genus $\left(M_{n}\right) \geq 2$ and that the induced morphisms $\pi_{1}\left(M_{n}\right) \rightarrow$ $\pi_{1}\left(N_{n}\right)$ between the fundamental groups are surjective. Then we can extract, up to dilatations, convergent subsequences of both the 
surfaces and the ambient manifolds to a compact stable surface weakly embedded in the limit manifold.

Moreover, the compactness is strong in the sense that the topological type of the surfaces and the affine diffeomorphism class of the ambient manifolds are preserved. We remark that for genus one surfaces the above compactness result is not true. For non compact ambient manifolds we can avoid the assumption on the fundamental groups and we can prove, see Corollary 4.5 , that

The moduli space of complete non compact orientable flat manifolds, identified by homotheties, which admit compact orientable embedded stable surfaces with genus greater than or equal to two is compact.

As the above moduli space is non compact, we conclude the non existence of this kind of surfaces for most of these flat three manifolds.

We point that an interesting family of compact orientable embedded stable surfaces are the boundaries of the solutions to the isoperimetric problem in complete orientable flat three manifolds, i.e., minimum area surfaces among the ones that enclose a fixed volume. Hence, a non existence result for some class of embedded stable surfaces will imply restrictions on the type of solutions to the isoperimetric problem. These solutions are not known, in general, for complete flat 3-manifolds, see $[R R]$ for the simplest case of flat 3-manifolds with cyclic fundamental group. Combining the above result on non compact flat three manifolds with the ones in $[\mathrm{RR}]$ we conclude that

For most of the 3-manifolds $T^{2} \times \mathbb{R}$, where $T^{2}$ is a flat two dimensional torus, the only solutions to the isoperimetric problem are geodesic balls, tubular neighborhoods of closed geodesics, and tubular neighborhoods of totally geodesic tori.

This paper is divided into four sections. In the first we recall some basic facts on complete orientable flat 3-manifolds and on embedded constant mean curvature surfaces in such ambient manifolds. In the second we state some basic results about complete orientable flat three manifolds and embedded constant mean curvature surfaces in such ambient manifolds, and we prove the convergence results needed in the next sections. In particular, we obtain a generalization of the classical Rolling theorem of Blaschke for surfaces with non negative mean curvature, Theorem 2.1, and a uniform local area bound for surfaces embedded in $\mathbb{R}^{3}$ with constant mean curvature invariant by a discrete subgroup $G$ of isometries of $\mathbb{R}^{3}$ such that $M / G$ is compact and orientable, see Theorem 2.5. In sections three and four we prove our compactness results and their consequences.

\section{Preliminaries}

Every complete flat three manifold is the quotient of $\mathbb{R}^{3}$ by a discrete subgroup $G$ of the group Iso $\left(\mathbb{R}^{3}\right)$ of affine isometries of $\mathbb{R}^{3}$ acting properly and discontinuously in $\mathbb{R}^{3}$. We are interested in orientable three manifolds and, since this fact is equivalent to the one that all the isometries in $G$ preserve the orientation of $\mathbb{R}^{3}$, we consider the set $\mathcal{G}$ of discrete orientation preserving subgroups of isometries acting properly and discontinuously in $\mathbb{R}^{3}$. For every subgroup $G \subset \mathcal{G}$ we shall denote by $\Gamma(G)$ the subgroup of translations in $G$ and by $\operatorname{inj}\left(\mathbb{R}^{3} / G\right)$ the injectivity radius of the manifold $\mathbb{R}^{3} / G$. The statements below follow directly from Wolf's results [Wo]. 


\section{Lemma 1.1.}

(i) If $G \in \mathcal{G}$, then the subgroup $\Gamma(G)$ is an abelian torsion free normal subgroup in $G$ with $\operatorname{rank}(\Gamma(G)) \leq 3$. $G$ is a cyclic subgroup if and only if $\operatorname{rank}(\Gamma(G))=0$ or 1. If $\operatorname{rank}(\Gamma(G))=2$, 3, then $\operatorname{order}(G / \Gamma(G)) \leq 6$.

(ii) The set $\{G \in \mathcal{G} ; \operatorname{rank}(\Gamma(G)) \geq 2\}$ contains only a finite number of affine conjugation classes.

(iii) Let $\left\{G_{n}\right\}_{n \in \mathbb{N}}, G \in \mathcal{G}$ be such that $G_{n} \rightarrow G$, i.e., $G$ is the set of accumulation points in $\operatorname{Iso}\left(\mathbb{R}^{3}\right)$ of the sequence $\left\{G_{n}\right\}_{n \in \mathbb{N}}$. If $\operatorname{rank}\left(\Gamma\left(G_{n}\right)\right) \geq 2$ then $\Gamma\left(G_{n}\right) \rightarrow$ $\Gamma(G)$ and $\operatorname{rank}(\Gamma(G)) \leq \liminf \operatorname{rank}\left(\Gamma\left(G_{n}\right)\right)$.

(iv) Let $\left\{G_{n}\right\}_{n \in \mathbb{N}}$ be contained in an affine conjugation class in $\mathcal{G}$, and $G \in \mathcal{G}$ such that $G_{n} \rightarrow G$. If $\operatorname{rank}(\Gamma(G))=\operatorname{rank}\left(\Gamma\left(G_{n}\right)\right)$ then $G$ lies in the above affine conjugation class. So there exists a sequence of affine transformations of $\mathbb{R}^{3}$ converging to the identity that induce diffeomorphisms between $\mathbb{R}^{3} / G_{n}$ and $\mathbb{R}^{3} / G$.

(v) Let $\left\{G_{n}\right\}_{n \in N} \subset \mathcal{G}$ be such that $\operatorname{inj}\left(\mathbb{R}^{3} / G_{n}\right)$ is bounded away from zero. Then we can extract a subsequence convergent to a subgroup $G \in \mathcal{G}$.

We describe briefly the elements $G \in \mathcal{G}$, up to affine conjugation, or, equivalently, the possible types of affinely diffeomorphic complete orientable flat three manifolds. See $[$ Wo] for details.

If $\operatorname{rank}(\Gamma(G))=0$ or 1 then either $G=\{\operatorname{Id}\}$ or $G=S_{\theta}$, with $0 \leq \theta \leq \pi$, where $S_{\theta}$ is the subgroup generated by a screw motion given by a rotation of angle $\theta$ followed by a non trivial translation in the direction of the rotation axis. Note that in this case the affine conjugation classes are parametrized by $\theta$.

If $\operatorname{rank}(\Gamma(G))=2$, then we have two affine conjugation classes: either $G$ is generated by two linearly independent translations and the quotient $\mathbb{R}^{3} / G$ is the Riemannian product $T^{2} \times \mathbb{R}$, where $T^{2}$ is a flat two dimensional torus, or $G$ is generated by a screw motion with angle $\pi$ and a translation orthogonal to the axis of the screw motion. We shall denote the class of quotient manifolds $\mathbb{R}^{3} / G$ by $\mathcal{K}$. Note that every manifold in $\mathcal{K}$ admits a twofold covering by some $T^{2} \times \mathbb{R}$.

The above manifolds describe all the possible types of affinely diffeomorphic complete non compact orientable flat three manifolds.

Finally, if $\operatorname{rank}(\Gamma(G))=3$, then either $G$ contains only translations and $\mathbb{R}^{3} / G$ is a flat three torus, or $G$ contains screw motions with angle different from 0 and $\mathbb{R}^{3} / \Gamma(G) \rightarrow \mathbb{R}^{3} / G$ is a Riemannian covering with at most six sheets. This kind of groups determines five different affine diffeomorphism classes.

Now we give a few restrictions to the existence of compact constant mean curvature surfaces embedded in orientable non compact flat three manifolds.

\section{Theorem 1.2.}

(i) The only compact connected constant mean curvature surface embedded in $\mathbb{R}^{3}$ is the round sphere.

(ii) The only compact connected constant mean curvature surfaces embedded in $\mathbb{R}^{3} / S_{\theta}$, with $0 \leq \theta \leq \pi$, are quotients of embedded $S_{\theta}$-invariant Delaunay surfaces.

(iii) A compact connected non totally geodesic constant mean curvature surface embedded in $T^{2} \times \mathbb{R}$ is orientable and symmetric with respect to a totally geodesic torus $T \times\{a\}$ for some $a \in \mathbb{R}$. 
We obtain (i) and (iii) by the Alexandrov reflection method [A]. In case (ii) the surface lies inside a right circular cylinder and the result follows from the sharper version of the above method by Korevaar, Kusner and Solomon [KKS].

Fixing the genus of the surface, we obtain other restrictions. We say that a surface $M$ embedded in a flat three manifold $N_{1}$ lifts to another flat three manifold $N_{2}$ if there exists a Riemannian covering $\Pi: N_{2} \rightarrow N_{1}$ and an isometric embedding $i: M \rightarrow N_{2}$ such that the composition $\Pi \circ i$ coincides with the inclusion $M \rightarrow N_{1}$. This is equivalent to the fact that $i_{*}\left(\pi_{1}(M)\right) \subset \Pi_{*}\left(\pi_{1}\left(N_{2}\right)\right)$.

Theorem 1.3. Let $M$ be an orientable compact connected constant mean curvature surface embedded in a complete orientable flat three manifold $N$. Then

(i) If genus $(M)=0$ then $M$ lifts to a round sphere in $\mathbb{R}^{3}$.

(ii) If genus $(M)=1$ then either $M$ is totally geodesic or $M$ lifts to a quotient of a Delaunay surface in $\mathbb{R}^{3} / S_{\theta}$.

Proof. In the genus zero case $M$ can be lifted to $\mathbb{R}^{3}$ and we conclude the result from Alexandrov's theorem [A]. If genus $(M)=1$ and $M$ is minimal then it follows from the Gauss-Bonnet theorem that $M$ is totally geodesic. If $M$ is not minimal then we consider a connected component $\widetilde{M}$ of the pullback of $M$ to the universal covering $\mathbb{R}^{3}$ of $N$. We have that $\widetilde{M}$ is properly embedded and covers $M$. Thus $\widetilde{M}$ must be, topologically, either a plane, a cylinder or a torus, and from the results in $[\mathrm{KKS}]$ it follows that $\widetilde{M}$ is a Delaunay surface.

\section{UNIFORM LOCAL AREA BOUNDS AND A CONVERGENCE RESULT}

Let $M \subset \mathbb{R}^{3}$ be a properly embedded not necessarily connected surface which is invariant by a subgroup $G \in \mathcal{G}$. Then the quotient $M / G$ is a Riemannian surface properly embedded in $\mathbb{R}^{3} / G$. We shall assume that $M / G$ is orientable, which is equivalent to assuming that the elements of $G$ are also orientation preserving when restricted to $M$. We choose an orientation on $M / G$ and we consider on $M$ the lifting of this orientation, which determines a Gauss map $\nu: M \rightarrow S^{2}$ which is $G$-equivariant.

The fact that $M / G$ separates $\mathbb{R}^{3} / G$ is equivalent to the fact that $\mathbb{R}^{3}-M$ is the disjoint union of two $G$-invariant open sets. Each one of these open sets, which are not necessarily connected, has boundary equal to $M$, and the $G$-equivariant normal vector $\nu$ to $M$ points towards one of them.

Since $M$ is the pullback of $M / G$ via the covering $\mathbb{R}^{3} \rightarrow \mathbb{R}^{3} / G$, the surface $M$ is connected if and only if the morphism between the fundamental groups $\pi_{1}(M / G) \rightarrow$ $\pi_{1}\left(\mathbb{R}^{3} / G\right)$ induced by the inclusion is surjective.

We start this section with the generalization of a result valid for convex surfaces. Blaschke's rolling theorem ensures that a sphere of radius $R>0$ can "roll" inside a convex surface whose principal curvatures are smaller than $1 / R$ and outside a convex surface whose principal curvatures are greater than $R$. If we replace the convexity hypothesis by the non negativity of the mean curvature we obtain a Blaschke type theorem at the side of the surface where the normal vector points

Theorem 2.1 (Rolling theorem for non negative mean curvature). Let $M \subset \mathbb{R}^{3}$ be a non totally geodesic embedded surface invariant by a subgroup $G \in \mathcal{G}$ such that $M / G$ is an orientable compact connected surface that separates $\mathbb{R}^{3} / G$. Let $\nu: M \rightarrow$ $S^{2}$ be the $G$-equivariant Gauss map and suppose that the mean curvature of $M$ is 
non negative. If we denote by

$$
c=c(M)=1 / \max \{\text { positive principal curvatures of } M\},
$$

and by $F: M \times[0, c) \rightarrow \mathbb{R}^{3}$ the normal exponential map on $M$, which is given by

$$
(p, t) \in M \times[0, c) \longmapsto p+t \nu(p),
$$

then we have that $F$ is a diffeomorphism onto its image.

Proof. Denote by $\psi: M \rightarrow \mathbb{R}^{3}$ the inclusion map. It is clear from the definition of $c$ and the compactness of $M / G$ that $0<c<\infty$, that $F$ is a local diffeomorphism and that the restriction of $F$ to $M \times[0, \varepsilon)$ is injective for $\varepsilon>0$ small enough.

For $0 \leq t<c$ the parallel surface determined by the immersion $\psi_{t}=F(-, t)$ from $M$ to $\mathbb{R}^{3}$ has mean curvature given by

$$
2 H_{t}=\frac{k_{1}}{1-t k_{1}}+\frac{k_{2}}{1-t k_{2}}=\frac{2 H-2 t K}{\left(1-t k_{1}\right)\left(1-t k_{2}\right)},
$$

where $k_{i}, H$ and $K$ are the principal curvatures, the mean curvature and the Gauss curvature of $\psi$. As $H^{2} \geq K$ with equality only at umbilic points, we have

$$
2 H_{t} \geq \frac{2 H(1-t H)}{\left(1-t k_{1}\right)\left(1-t k_{2}\right)} \geq 0 .
$$

Moreover, $H_{t}=0$ at some point implies that the point is flat for the embedding $\psi$.

Let $t_{0}=\sup \left\{t>0 ; F: M \times[0, t] \rightarrow \mathbb{R}^{3}\right.$ is injective $\}$. We are going to prove the theorem by contradiction. Let us suppose that $t_{0}<c$. Then there exist two distinct points in $M \times\left[0, t_{0}\right]$ with the same image under $F$. These points are necessarily on the boundary of $M \times\left[0, t_{0}\right]$ and, since $M / G$ is embedded and separates $\mathbb{R}^{3} / G$, they have to be at $M \times\left\{t_{0}\right\}$.

So suppose that $F\left(p, t_{0}\right)=F\left(q, t_{0}\right)$ for two distinct points $p$ and $q$. Then the immersion $\psi_{t_{0}}$ has a tangential contact at those points. As the normal vector to an immersion coincides with those of its parallel surfaces, we conclude that $\nu(p)$ and $\nu(q)$ are proportional. If $\nu(p)=\nu(q)$ then it follows from the definition of $F$ that $\psi(p)=\psi(q)$, which is not possible since $\psi$ is an embedding. So $\nu(p)=-\nu(q)$ and, as $H_{t_{0}} \geq 0$, an application of the maximum principle [Sc] shows that there exist neighborhoods of $p$ and $q$ in $M$ with $H_{t_{0}} \equiv 0$ which have the same image under the immersion $\psi_{t_{0}}$. This implies that $\psi$ is totally geodesic on these neighborhoods and, by a connectedness argument on $M / G$ and the $G$-invariance of $M$, we have that $\sigma \equiv 0$ on $M$, which contradicts our hypothesis on $M$. This proves the theorem.

Corollary 2.2. With the same hypothesis of Theorem 2.1, if genus $(M / G) \neq 1$ and $G$ is not cyclic then $\operatorname{inj}\left(\mathbb{R}^{3} / G\right) \geq \frac{c(M)}{6}$.

Proof. Since $G$ is not cyclic we have that $\operatorname{order}(G / \Gamma(G)) \leq 6$ from Lemma 1.1, and we conclude that $M / \Gamma(G)$ is a compact surface with genus different from one. Since the Gauss map $\nu$ on $M$ can be induced on the quotient surface $M / \Gamma(G)$, it follows from the Gauss-Bonnet theorem that $\nu$ is surjective. Now let $v \in \Gamma(G)-\{0\}$. Take a point $p \in M$ such that the normal vector $\nu(p)$ equals $v /|v|$. As $M$ is invariant by the translation by the vector $v$, then $q=p+v$ lies in $M$ and so $F(p,|v|)=F(q, 0)$. We obtain from Theorem 2.1 that $|v| \geq c$, and this implies that $\operatorname{inj}\left(\mathbb{R}^{3} / \Gamma(G)\right) \geq c$. It follows from a standard argument that $\operatorname{inj}\left(\mathbb{R}^{3} / G\right) \geq \frac{c}{d}$, where $d \leq 6$ is the number of sheets of the covering $\mathbb{R}^{3} / \Gamma(G) \rightarrow \mathbb{R}^{3} / G$, and we have proved the theorem. 
We wish to apply the rolling Theorem 2.1 when the mean curvature of $M$ is constant. In this case the separation assumption follows from the other hypotheses, as proved in next lemma

Lemma 2.3. A compact connected orientable non totally geodesic constant mean curvature surface embedded in a complete orientable flat three manifold separates the ambient manifold in two connected components.

Proof. Let $\mathbb{R}^{3} / G$, with $G \in \mathcal{G}$, be a complete orientable flat three manifold, and $\widetilde{M} \subset \mathbb{R}^{3} / G$ an embedded orientable compact connected not totally geodesic constant mean curvature surface. Let $M$ be the pullback of $\widetilde{M}$ by the covering map $\mathbb{R}^{3} \rightarrow \mathbb{R}^{3} / G$.

Let us suppose that $\mathbb{R}^{3} / G-\widetilde{M}$ is connected. Then there exists a simple closed curve $\gamma:[0, \ell] \rightarrow \mathbb{R}^{3} / G$ that intersects $\widetilde{M}$ orthogonally at a single point, and so the intersection number $\#(\widetilde{M} \cap \gamma)$ of $\widetilde{M}$ and $\gamma$ is \pm 1 . The curve $\gamma$ cannot be homotopic, in $\mathbb{R}^{3} / G$, to a curve contained in $\widetilde{M}$, since then $\#(\widetilde{M} \cap \gamma)=0$. In particular, the morphism induced between the fundamental groups by the inclusion $\widetilde{M} \subset \mathbb{R}^{3} / G$ is not surjective. It follows that $M$ is not connected. More precisely, if $\alpha$ is a lifting of $\gamma$ to $\mathbb{R}^{3}$ origin lies in a connected component $M_{1}$ of $M$, then its extreme point lies in another connected component $M_{2}$ of $M$.

As $M_{1}$ and $M_{2}$ separate $\mathbb{R}^{3}$, there is exactly one connected component $\Omega$ of $\mathbb{R}^{3}-\left(M_{1} \cup M_{2}\right)$ whose boundary is $M_{1} \cup M_{2}$. The curve $\alpha$ lies in $\Omega$ because it can only intersect $M$ at its extreme points. As $\widetilde{M}$ is orientable, the induced orientation on $M$ determines a $G$-equivariant Gauss map on $M, \nu: M \rightarrow S^{2}$, and we can suppose, after changing the orientation of $\gamma$ if neccesary, that $\alpha^{\prime}(0)=\nu(\alpha(0))$. Since $\nu$ is $G$-equivariant we obtain that $\alpha^{\prime}(\ell)=\nu(\alpha(\ell))$, and so the normal vector $\nu$ points towards $\Omega$ on $M_{1}$ and out of $\Omega$ on $M_{2}$.

Let $p_{1} \in M_{1}, p_{2} \in M_{2}$ be points of minimum distance; these exist by the periodicity of the surfaces and the compactness of $\widetilde{M}$. The minimizing geodesic joining $p_{1}$ and $p_{2}$ lies in $\Omega$, and the surfaces $M_{2}$ and $\left(p_{2}-p_{1}\right)+M_{1}$ meet tangentially at $p_{2}$ with the same normal vector $\nu$ and the same constant mean curvature. It follows from the maximum principle that $\left(p_{2}-p_{1}\right)+M_{1}=M_{2}$, and so the pair of points $q \in M_{1}, q^{\prime}=q+\left(p_{2}-p_{1}\right) \in M_{2}$ are points of minimum distance. Hence the normal vectors to $M_{1}$ and $M_{2}$ are proportional to $p_{2}-p_{1}$, and $M_{1}$ and $M_{2}$ are parallel planes in $\mathbb{R}^{3}$. As this contradicts our hypothesis on $M$, the lemma is proved.

For minimal surfaces we obtain a better result

Corollary 2.4 (Rolling theorem for minimal surfaces). Let $M \subset \mathbb{R}^{3}$ be a non totally geodesic embedded minimal surface invariant by a subgroup $G \in \mathcal{G}$ such that $M / G$ is an orientable compact surface. Then $M$ has an embedded tubular neighborhood of radius

$$
c=c(M)=1 / \max \{\text { positive principal curvatures of } M\},
$$

that is, the normal exponential map $F: M \times(-c, c) \rightarrow \mathbb{R}^{3}$ is a diffeomorphism onto its image.

Proof. From the minimality of $M$ and the compactness of $M / G$, we conclude using the maximum principle that $M$ is connected, and so $M / G$ is connected. The orientability of $M / G$ and the connectedness of $M$ imply that $M / G$ separates $\mathbb{R}^{3} / G$. 
So, if $\nu: M \rightarrow S^{2}$ is a $G$-equivariant Gauss map, both $\nu$ and $-\nu$ satisfy the hypothesis of Theorem 2.1 and, since the "half tubular" neighborhoods are contained in different $G$-invariant components of $\mathbb{R}^{3}-M$, the proof of the corollary follows easily.

Theorem 2.5 (Local area bound). Let $M \subset \mathbb{R}^{3}$ be a non totally geodesic embedded surface with non negative mean curvature invariant by a subgroup $G \in \mathcal{G}$ and such that $M / G$ is an orientable compact surface that separates $\mathbb{R}^{3} / G$. If the second fundamental form of $M$ is uniformly bounded, $|\sigma|<C$, then, for any $R$ with $0<$ $R<\frac{1}{C}$, we have

$$
\operatorname{area}\left(M_{R}\right) \leq 36 \pi R^{2}
$$

where $B_{R}$ is any Euclidean ball of radius $R$ and $M_{R}=M \cap B_{R}$.

Proof. Consider the normal exponential map $F: M \times[0, c(M)) \rightarrow \mathbb{R}^{3}$, which is a diffeomorphism onto its image by Theorem 2.1. It follows easily that $\frac{R}{2}<c(M)$, and so $F$ maps $M_{R} \times\left[0, \frac{R}{2}\right)$ injectively over a region $\Omega$ in $\mathbb{R}^{3}$. By the change of variables formula

$$
\operatorname{vol}(\Omega)=\int_{0}^{R / 2}\left\{\int_{M_{R}}\left(1-t k_{1}\right)\left(1-t k_{2}\right) d A\right\} d t,
$$

where $k_{i}$ are the principal curvatures of $M$ and $\left(1-t k_{1}\right)\left(1-t k_{2}\right)$ is the Jacobian of the map $F$. Since $0 \leq t \leq \frac{R}{2}$ and $k_{1}, k_{2} \leq C$, we have that the volume of $\Omega$ is greater than or equal to

$$
\int_{0}^{R / 2}\left\{\int_{M_{R}}\left(1-\frac{R}{2} C\right)^{2} d A\right\} d t
$$

As $\frac{R}{2} C \leq \frac{1}{2}$, the last integral is greater than or equal to

$$
\int_{0}^{R / 2} \int_{M_{R}} \frac{1}{4} d A=\frac{R}{8} \operatorname{area}\left(M_{R}\right)
$$

and we conclude that $\operatorname{vol}(\Omega) \geq \frac{R}{8}$ area $\left(M_{R}\right)$.

Now the theorem follows from the fact that $\Omega$ is contained in a Euclidean ball with radius $\frac{3}{2} R$ and so $\operatorname{vol}(\Omega) \leq \frac{9}{2} \pi R^{3}$. To see this, take $p \in \Omega$. Then $d\left(p, M_{R}\right)<$ $\frac{R}{2}$, and so the distance between the center of $B_{R}$ and $p$ is less than $\frac{3}{2} R$ as we claimed.

Remark. Using the same idea we can estimate the volume of the region $\Omega$ in $\mathbb{R}^{3} / G$ $M / G$ which is the projection of the $G$-invariant region of $\mathbb{R}^{3}-M$ where the mean curvature vector points, and we obtain

$$
\begin{aligned}
\operatorname{vol}(\Omega) & \geq \int_{0}^{c(M)}\left\{\int_{M / G}\left(1-t k_{1}\right)\left(1-t k_{2}\right) d A\right\} d t \\
& =c(M) \operatorname{area}(M / G)-c(M)^{2} \int_{M / G} H d A+\frac{c(M)^{3}}{3} 2 \pi \chi(M / G) .
\end{aligned}
$$

The following basic convergence result can be found in $[\mathrm{KKS}]$ or $[\mathrm{W}]$

Theorem 2.6. Let $M_{n} \subset \mathbb{R}^{3}$ be a sequence of properly embedded surfaces with constant mean curvature such that $\left|\sigma_{n}\right|<C$ for all $n \in \mathbb{N}$ which satisfy uniform local area bounds. Then either the sequence $M_{n}$ has no limit points or we can 
extract a subsequence convergent in the $C^{k}$ topology (for all $k \in \mathbb{N}$ ) to a properly weakly embedded constant mean curvature surface $M$.

We say that $\left\{M_{n}\right\}_{n \in \mathbb{N}}$ satisfy uniform local area bounds if there is a radius $r>0$ such that for each ball $B_{r} \subset \mathbb{R}^{3}$ we have that area $\left(M_{n} \cap B_{r}\right)<C^{\prime}$, with $C^{\prime}$ independent of $n$.

The $C^{k}$ convergence means that near any point $p \in M$ and for $n$ large enough each $M_{n}$ is locally a graph over a fixed disc $D \subset T_{p} M$ and the graphs of $M_{n}$ converge in the usual $C^{k}$ topology to the graph of $M$. We say that an immersed surface $M \subset \mathbb{R}^{3}$ is weakly embedded if it only holds tangential self-intersections.

Recall from Lemma 1.1 and Theorem 1.2 that if a flat 3 -manifold $\mathbb{R}^{3} / G$, with $G \in \mathcal{G}$, admits an embedded compact orientable constant mean curvature of genus greater than one, then $\operatorname{rank}(\Gamma(G)) \geq 2$ or, equivalently, $G$ is not cyclic. In particular, such groups $G$ determine a finite number of affine conjugation classes. Denote by $\mathcal{A}$ one of these affine conjugation classes. We shall need the following lemma

Lemma 2.7. Consider a sequence $\left\{G_{n}\right\}_{n \in \mathbb{N}}$ in $\mathcal{A}$. Take a sequence of connected embedded surfaces $\left\{M_{n}\right\}_{n \in \mathbb{N}}$ in $\mathbb{R}^{3}$ such that the surface $M_{n}$ is $G_{n}$ invariant for all $n \in \mathbb{N}$ and $M_{n} / G_{n}$ are compact surfaces. Suppose that $G_{n}$ converge to $G$ in $\mathcal{G}$ and that $M_{n}$ converge to a properly immersed $G$-invariant surface $M$ in $\mathbb{R}^{3}$. If $M / G$ is a compact surface, then $G \in \mathcal{A}$ and $\mathbb{R}^{3} / G_{n}$ is affinely diffeomorphic to $\mathbb{R}^{3} / G$ for $n$ large.

Proof. Put $\Gamma=\Gamma(G)$. From Lemma 1.1 we have that $\Gamma\left(G_{n}\right) \rightarrow \Gamma$ and, since we have that $\operatorname{order}\left(G_{n} / \Gamma\left(G_{n}\right)\right) \leq 6$, it follows that $\operatorname{order}(G / \Gamma) \leq 6$ and so $M / \Gamma$ is compact.

By Lemma 1.1 it is enough to prove that $\operatorname{rank}(\Gamma)=\operatorname{rank}\left(\Gamma\left(G_{n}\right)\right)$. Suppose, reasoning by contradiction, that $\operatorname{rank}(\Gamma)<\operatorname{rank}\left(\Gamma\left(G_{n}\right)\right)$.

Let $\left\{w^{i}\right\}_{i \in I}$ be a basis of $\Gamma$ and $V$ the vector space in $\mathbb{R}^{3}$ generated by $\left\{w^{i}\right\}_{i \in I}$. We have that $\operatorname{dim}(V)<\operatorname{rank}\left(\Gamma\left(G_{n}\right)\right) \leq 3$. Take vectors $w_{n}^{i} \in \Gamma\left(G_{n}\right)$ that converge the vectors $w^{i}$ and call $V_{n}$ the vector space generated by $\left\{w_{n}^{i}\right\}_{i \in I}$. Note that these vectors generate a discrete subgroup $\Gamma_{n}^{\prime}$ such that $V_{n} / \Gamma_{n}^{\prime}$ is compact. Let $S_{n}(r)$, with $r>0$, be the sphere centered on the origin of radius $r$ in the orthogonal subspace $V_{n}^{\perp}$ to $V_{n}$. Suppose that $M$ contains the origin of $\mathbb{R}^{3}$. Since $\operatorname{rank}\left(\Gamma\left(G_{n}\right)\right)>$ $\operatorname{dim}\left(V_{n}\right)$, we have that $\Gamma\left(G_{n}\right) \not \subset V_{n}$ and so, by the connectedness and the $\Gamma\left(G_{n}\right)$ invariance of $M_{n}$, we conclude that the surfaces $M_{n}$ meet $S_{n}(r) \times V_{n}$ for every $r>0$.

Moving these points by the vectors in $\Gamma_{n}^{\prime}$ and using the compactness of $V_{n} / \Gamma_{n}^{\prime}$ and the fact that $\Gamma_{n}^{\prime} \rightarrow \Gamma$, we obtain that the surface $M$ meets $S(r) \times V$ for all $r>0$, where $S(r)$ is the sphere of radius $r$ in $V^{\perp}$. Then $M / \Gamma$ meets all the surfaces $S(r) \times(V / \Gamma)$ and then it is necessarily non compact, and this contradiction proves the lemma.

Theorem 2.8. Let $\left\{M_{n}\right\}_{n \in \mathbb{N}}$ be a sequence of constant mean curvature surfaces embedded in $\mathbb{R}^{3}$ invariant by discrete subgroups $\left\{G_{n}\right\}_{n \in N} \subset \mathcal{A}$, where $\mathcal{A}$ is an affine conjugation class in $\mathcal{G}$ whose elements are not cyclic. Suppose that $M_{n} / G_{n}$ are compact connected surfaces with genus greater than one and second fundamental forms uniformly bounded, $\left|\sigma_{n}\right|<C$. 
Then we can extract a subsequence of $G_{n}$ convergent to a subgroup $G \in \mathcal{G}$ and, if the sequence $M_{n}$ has an accumulation point, we can extract a subsequence convergent in the $C^{k}$ topology, $k \geq 2$, to a $G$-invariant properly weakly embedded constant mean curvature surface $M$. Moreover

(i) If the multiplicity of $M$ is greater than one then $M$ is an embedded minimal surface with multiplicity two.

(ii) If $M / G$ is compact and the surfaces $M_{n}$ are connected then the multiplicity of $M$ is one, the $M_{n} / G_{n}$ are diffeomorphic to $M / G$ for $n$ large enough, and $\mathbb{R}^{3} / G_{n}$ and $\mathbb{R}^{3} / G$ are affinely diffeomorphic.

(iii) If the surfaces $M_{n}$ are minimal then $M$ is embedded with multiplicity one.

Proof. We know by Theorem 2.5 that if $\left\{M_{n}\right\}_{n \in \mathbb{N}}$ is a sequence in the conditions of the theorem, the uniform curvature estimate $\left|\sigma_{n}\right|<C$ implies a uniform local area bound for the sequence. So we can extract a subsequence of $M_{n}$ convergent to a constant mean curvature surface $M$ which is weakly embedded in $\mathbb{R}^{3}$. Since the injectivity radii $\operatorname{inj}\left(\mathbb{R}^{3} / G_{n}\right)$ are greater than $\frac{1}{6 C}$ by Corollary 2.2 , we can also extract a subsequence of the subgroups $\left\{G_{n}\right\}_{n \in \mathbb{N}}$ convergent to a subgroup $G \in \mathcal{G}$, by lemma 1.1 .

Let $p \in M$. Since $M$ is a limit of embedded surfaces, it can only have tangential contacts and so the intersection of $M$ with a ball $B$ of small enough radius centered at $p$ is the union of either one or several graphs meeting tangentially at $p$. Each one of these graphs is the limit of one or several components of $M_{n} \cap B$. As all the surfaces $M_{n}$ admit by Theorem 2.1 a "half tubular" neighborhood of radius $1 / C$ on the side where the normal vector points, then $M_{n} \cap B$ has at most two connected components. From the $C^{k}$ convergence of $M_{n}$ to the surface $M$ we deduce that either $M \cap B$ consists of two different sheets meeting tangentially at $p$ (by the maximum principle this only happens when the limit surface $M$ is not minimal), or $M \cap B$ is embedded and is the limit of one or two components of $M_{n} \cap B$. If $M \cap B$ is the limit of two components of $M_{n} \cap B$, these induce on $M \cap B$ opposite normal vectors fields and so the mean curvature of $M \cap B$ must be invariant by a change of the normal, i.e., the limit surface $M$ is minimal. This proves (i).

Now we show (ii). Suppose, reasoning by contradiction, that the multiplicity of $M / G$ is two. By (i) the surface $M / G$ is minimal. By Lemma 2.7 we have that the limit manifold $\mathbb{R}^{3} / G$ is affinely diffeomorphic to $\mathbb{R}^{3} / G_{n}$ and so, by Lemma 1.1 , we can find a sequence of affine transformations of $\mathbb{R}^{3}$ converging to the identity which induce diffeomorphisms $f_{n}: \mathbb{R}^{3} / G_{n} \rightarrow \mathbb{R}^{3} / G$. For $n$ large enough, using the compactness of the surfaces $M_{n} / G_{n}$ and $M / G$, we have twofold coverings $\Pi_{n}$ : $M_{n} / G_{n} \rightarrow M / G$ that induce over the points of $M / G$ opposite orientations, which implies that $M / G$ is non orientable, and such that the two maps

$$
\begin{aligned}
& M_{n} / G_{n} \stackrel{\Pi_{n}}{\rightarrow} M / G \rightarrow \mathbb{R}^{3} / G, \\
& M_{n} / G_{n} \rightarrow \mathbb{R}^{3} / G_{n} \stackrel{f_{n}}{\rightarrow} \mathbb{R}^{3} / G
\end{aligned}
$$

determine the same morphism between the fundamental groups. The second map induces a surjective morphism between the fundamental groups of $M_{n} / G_{n}$ and $\mathbb{R}^{3} / G$ by hypothesis. The first is topologically equivalent to the composition of the projection of the orientable double cover of $M / G$ with the embedding $M / G \rightarrow$ $\mathbb{R}^{3} / G$, which never induces a surjective morphism between the fundamental groups (otherwise, taking pullback to $\mathbb{R}^{3}$, we would obtain that the orientation double 
covering of $M$ would be connected, which is impossible because $M$ is properly embedded in $\mathbb{R}^{3}$ ). This contradiction proves that the multiplicity of $M$ is one. Since $M_{n} / G_{n} \rightarrow M / G$ with multiplicity one and all the surfaces are compact, it follows easily that $M_{n} / G_{n}$ and $M / G$ are diffeomorphic. This completes the proof of (ii).

If all the surfaces $M_{n}$ are minimal then Corollary 2.4 shows that there exists a tubular neighborhood of radius $1 / C$ for each $M_{n}$, and so $M \cap B$ is embedded and is the limit of one sequence of components of $M_{n} \cap B$, which proves (iii).

Remark. The arguments in the proof of (ii) show the following: if $G \in \mathcal{A}$ and the limit surface $M / G$ is compact with multiplicity two, then $M / G$ is a non orientable minimal surface. Of course in this case the surfaces $M_{n}$ are non connected.

Note that, in general, for the limits obtained in the above theorem neither the topological type of the surfaces $M_{n} / G_{n}$ nor the affine diffeomorphism class of the ambient manifolds $\mathbb{R}^{3} / G_{n}$ is preserved.

\section{INDEX ONE MINIMAL SURFACES}

The purpose of this section is to prove the compactness of the space of embedded triply periodic minimal surfaces that induce index one minimal surfaces in a non fixed three torus. For simply and doubly periodic minimal surfaces this compactness result it is not true. In other flat three manifolds the problem is still open. We recall that Ross [Ro] has proved that Schwarz's $\mathcal{P}$ and $\mathcal{D}$ triply periodic minimal surfaces have this property. It is also known that the genus of an orientable index one minimal surface in a three torus must be 3,4 or 5 , see for example $[R R]$. In the non periodic case López and Ros [LR] have proved that the catenoid and Enneper surface are the only complete orientable minimal surfaces in $\mathbb{R}^{3}$ with index one. By using similar methods we can classify the embedded index one minimal surfaces in the simply and doubly periodic case. It is a remarkable fact that these families are non compact. This classification will be used in the proof of the compactness theorem for the family of index one triply periodic minimal surfaces.

\section{Proposition 3.1.}

(i) An orientable minimal surface properly embedded in $\mathbb{R}^{3} / T_{v}$, where $T_{v}$ is the subgroup generated by a non trivial translation, has index one if and only if $M$ is either a helicoid or lies in the one parameter family of simply periodic Scherk surfaces.

(ii) An orientable minimal surface properly embedded in $T^{2} \times \mathbb{R}$, where $T^{2}$ is a flat bidimensional torus, has index one if and only if $M$ is an orientable doubly periodic Scherk surface.

Remark. The simply and doubly periodic Scherk surfaces are one parameter families of genus zero surfaces with total curvature $-4 \pi$ and four ends asymptotic to flat vertical annuli. The latter only exists when $T^{2}$ is a rhomboidal torus. It is proved in $[\mathrm{HM}]$ and $[\mathrm{MR} 1]$ that the surfaces in the statement of the proposition are the only orientable properly embedded minimal surfaces with total curvature $-4 \pi$ in $\mathbb{R}^{3} / T_{v}$ and $T^{2} \times \mathbb{R}$, respectively.

Proof of the proposition. We know by the work of Fischer-Colbrie $[\mathrm{FC}]$ that an orientable minimal surface in a flat orientable three manifold with finite index has finite total curvature, and so it is conformally equivalent to a compact Riemann 
surface $\bar{M}$ of genus $g$ minus a finite number of points $\left\{p_{1}, \ldots, p_{r}\right\}$. Moreover, if $M$ has a Gauss map $\nu$ then $\nu$ extends smoothly to $\bar{M}$ and the index of $M$ is given by the one of the operator $\Delta+|\nabla \nu|^{2}$ on $\bar{M}$, where the Laplacian and the modulus of the gradient of $\nu$ are computed in any metric compatible with the conformal structure of $\bar{M}$. On such a Riemann surface it is always possible to find a meromorphic map $\phi$ with degree less than or equal to $1+\left[\frac{g+1}{2}\right]$, see $[\mathrm{GH}]$, where $[x]$ is the least integer greater than or equal to $x$. It is also known that on a minimal surface with index one and Gauss map $\nu$ the degree of $\nu$ is less than or equal to the degree of any other meromorphic map on $\bar{M}$, see $[\mathrm{MoR}]$. Hence

$$
-\int_{M} K=4 \pi \operatorname{degree}(\nu) \leq 4 \pi\left(1+\left[\frac{1+g}{2}\right]\right) .
$$

If $M \subset T^{2} \times \mathbb{R}$ is a properly embedded minimal surface, it has been proved by Meeks and Rosenberg [MR1] that $\int_{M} K=2 \pi(2-g-r)$ and that $M$ has at least four ends. Hence we obtain from (2) that $2 g+2 \leq 2(1+[(1+g) / 2])$ and either $g=0$ or $g=1$. The case $g=1$ is not possible because there are no index one minimal surfaces with Gauss map and genus one, see [MoR]. If $g=0$ the number of ends must be four, the degree of the Gauss map is one, and so we have that $\int_{M} K=-4 \pi$.

If $M \subset \mathbb{R}^{3} / T_{v}$ is a properly embedded minimal surface we know that $\int_{M} K=$ $2 \pi(2-2 g-r-W(M))$, where $W(M) \geq 0$ is the total winding number of $M$, and that the number of ends is even [MR2, th.8]. So we have that $2 g \leq 2(1+[(1+g) / 2])$ and then $g=0,1,2$, or 3 . The case $g=1$ is not possible as before. If $g=2,3$ we obtain that $r=2$ and $W(M)=0$, that is, $M$ has two Scherk type ends. But such a surface cannot exist because the pullback of $M$ would be a properly embedded minimal surface in $\mathbb{R}^{3}$ contained in a halfspace, see [HM1]. If $g=0$ we have that $\int_{M} K=-4 \pi$.

Conversely, given a minimal surface in $\mathbb{R}^{3} / T_{v}$ or $T^{2} \times \mathbb{R}$ with total curvature $-4 \pi$, its Gauss map has degree one, and it is easy to see that this surface must have index one, see $[\mathrm{MoR}]$.

We now prove the main result in this section

Theorem 3.2 (Compactness of the space of index one minimal surfaces). Let a sequence of minimal surfaces $\left\{M_{n}\right\}_{n \in \mathbb{N}}$ be properly embedded in $\mathbb{R}^{3}$ invariant by lattices $\Gamma_{u}$ (i.e., rank three subgroups of translations in $\mathbb{R}^{3}$ ), normalized in such a way that the injectivity radius of $\mathbb{R}^{3} / \Gamma_{n}$ is one. Suppose that $M_{n} / \Gamma_{n}$ is an orientable compact surface with index one in $\mathbb{R}^{3} / \Gamma_{n}$. Then

(i) We can extract a convergent subsequence of $\Gamma_{n}$ to a lattice $\Gamma$.

(ii) We can extract a subsequence, denoted in the same way, of $M_{n}$ converging in the $C^{k}$ topology, $k \geq 2$, to an embedded triply periodic $\Gamma$-invariant minimal surface $M$. In particular, the surfaces $M_{n} / \Gamma_{n}$ and $M / \Gamma$ are diffeomorphic and $M_{n} / \Gamma_{n} \rightarrow M / \Gamma$.

Proof. The compact surfaces $M_{n} / \Gamma_{n}$ are non flat because they have index one, and so they are necessarily connected. As the topological type of $M_{n} / \Gamma_{n}$ is less than or equal to five we can suppose that their genus is constant. It is well known that this genus must be greater than or equal to three. 
We first prove that $\left|\sigma_{n}\right|<C$ for some constant $C>0$. If not, there would exist a sequence $p_{n} \in M_{n}$ such that $\lambda_{n}=\max \left|\sigma_{n}\right|_{p_{n} \in M_{n}}=\left|\sigma_{n}\right|\left(p_{n}\right) \rightarrow \infty$. By moving the points $p_{n}$ to the origin and expanding the surfaces $M_{n}$ by a factor $\lambda_{n}$ we obtain a sequence of embedded minimal surfaces $\widetilde{M}_{n}$ such that $\left|\widetilde{\sigma}_{n}\right|(0)=1$ and $\left|\widetilde{\sigma}_{n}\right| \leq 1$. By Corollary 2.8, a subsequence of $\widetilde{M}_{n}$, denoted in the same way, converges in the $C^{k}$ topology to a non flat properly embedded minimal surface $\widetilde{M}$ in $\mathbb{R}^{3}$. By our assumption on the injectivity radius of $\mathbb{R}^{3} / \Gamma_{n}$ (which coincides with the minimum length of the non zero vectors in $\Gamma_{n}$ ) it follows that the only accumulation point of the lattices $\lambda_{n} \Gamma_{n}$ is the vector 0 .

Since $\widetilde{M}$ is not totally geodesic it must be connected. Compact regions in $\widetilde{M}$ can be uniformly approximated by regions in $\widetilde{M}_{n} / \lambda_{n} \Gamma_{n}$, and so the index of the surface $\widetilde{M} \subset \mathbb{R}^{3}$ is less than or equal to one. As $\operatorname{Ind}(\widetilde{M})=0$ would imply that $\widetilde{M}$ is totally geodesic, we conclude that $\operatorname{Ind}(\widetilde{M})=1$ and so $\widetilde{M}$ is the catenoid, see [LR]. Since genus $\left(M_{n} / \Gamma_{n}\right) \geq 3$, it follows that the total curvature of $M_{n} / \Gamma_{n}$ is less than or equal to $-8 \pi$. As the total curvature of $\widetilde{M}$ is $-4 \pi$ we contradict Assertion 3.1 below, and so we conclude that the sequence $\left|\sigma_{n}\right|$ is uniformly bounded.

Assertion 3.1. The total curvature of $M_{n} / \Gamma_{n}$ is the same as that of $\widetilde{M}$.

The surface $\widetilde{M}$ is conformally the Riemann sphere $\Sigma$ minus two points $p_{1}, p_{2}$. Let $\left(D_{i}, z_{i}\right)$ be conformal disks on $\Sigma$ centered on $p_{i}$. Fix $\varepsilon>0$ and let $\Omega(\varepsilon)$ be the bounded domain $\Sigma-\bigcup_{i=1}^{2}\left\{z_{i} \in D_{i} ;\left|z_{i}\right|<\varepsilon\right\}$. We can choose $\varepsilon$ small enough so that $\operatorname{Ind}(\Omega(\varepsilon))=1$. We consider on the bounded domain $\Omega\left(\varepsilon^{2}\right)$, which contains $\Omega(\varepsilon)$, the function $u_{\varepsilon}$ defined by

$$
u_{\varepsilon}= \begin{cases}0, & \text { on } \Omega(\varepsilon), \\ \frac{\log \left(\left|z_{i}\right| / \varepsilon\right)}{\log (\varepsilon)}, & \text { on } z_{i} \in D_{i}, \quad \varepsilon^{2} \leq\left|z_{i}\right| \leq \varepsilon .\end{cases}
$$

It is well known that the energy of $u_{\varepsilon}$ goes to 0 when $\varepsilon \rightarrow 0$.

Since $\Omega\left(\varepsilon^{2}\right)$ can be uniformly approximated by regions in $\widetilde{M}_{n} / \lambda_{n} \Gamma_{n}$ and both the index of $I$ and the Dirichlet integral are invariant by scaling, we can find on the surfaces $M_{n} / \Gamma_{n}$, for $n$ large enough depending on $\varepsilon$, a domain $A_{n}$, a subdomain $A_{n}^{\prime} \subset A_{n}$ and a function $u_{n}$ defined on $A_{n}$ with $u_{n}=0$ on $A_{n}^{\prime}, u_{n}=1$ on $\partial A_{n}$, such that, after scaling by $\lambda_{n}$, the objects $A_{n}, A_{n}^{\prime}$ and $u_{n}$ are uniformly close to $\Omega\left(\varepsilon^{2}\right)$, $\Omega(\varepsilon)$ and $u_{\varepsilon}$, respectively. Moreover, we can suppose that $\operatorname{Ind}\left(A_{n}^{\prime}\right)=1$, and that the total energy of $u_{\varepsilon}$ is $O_{1}(\varepsilon)$, where $O_{i}(\varepsilon)$ will denote a quantity going to zero as $\varepsilon \rightarrow 0$. The function $u_{n}$ can be extended on $M_{n} / \Gamma_{n}$ by 1 outside $A_{n}$ to obtain a continuous Lipschitz function $v_{n}$ with support contained in the complement of $A_{n}$. As the indexes of $M_{n} / \Gamma_{n}$ and $A_{n}^{\prime}$ are both one, we conclude that $\operatorname{Ind}\left(M_{n} / \Gamma_{n}-A_{n}^{\prime}\right)=0$. In particular, since $\operatorname{supp}\left(v_{n}\right)$ is contained in $M_{n} / \Gamma_{n}-A_{n}^{\prime}$, the index form applied to $v_{n}$ must be non negative and so

$$
\begin{aligned}
0 & \leq \int_{M_{n} / \Gamma_{n}}\left\{\left|\nabla v_{n}\right|_{n}^{2}+2 K_{n} v_{n}^{2}\right\} \\
& \leq \int_{M_{n} / \Gamma_{n}}\left|\nabla u_{n}\right|^{2}+2 \int_{\left\{v_{n} \equiv 1\right\}} K_{n} \\
& =O_{1}(\varepsilon)+2 \int_{M_{n} / \Gamma_{n}} K_{n}-2 \int_{A_{n}} K_{n}
\end{aligned}
$$


where $K_{n}$ is the Gauss curvature of $M_{n}$. As the finite total curvature of $\widetilde{M}$ is close to that of $\Omega\left(\varepsilon^{2}\right)$, we have that $\int_{\widetilde{M}} \widetilde{K}=\int_{A_{n}} K_{n}+O_{2}(\varepsilon)$, where $\widetilde{K}$ is the Gauss curvature of $\widetilde{M}$. Combining this formula with the above inequality, we conclude that

$$
\int_{M_{n} / \Gamma_{n}}\left|K_{n}\right| \leq \int_{\widetilde{M}}|\widetilde{K}|+O_{3}(\varepsilon) .
$$

As the two integrals in the above inequality are independent of $n$ and $\varepsilon$, it follows that

$$
\int_{M_{n} / \Gamma_{n}}\left|K_{n}\right| \leq \int_{\widetilde{M}}|\widetilde{K}|
$$

The converse inequality is obtained from the lower semicontinuity of the absolute total curvature for the $C^{k}$ topology, $k \geq 2$. This proves the assertion.

By our normalization the lattices $\Gamma_{n}$ do not contain non zero vectors with length less than one, and so a subsequence, denoted in the same way, converges in the Euclidean group Iso $\left(\mathbb{R}^{3}\right)$ to a discrete subgroup $\Gamma$ of translations, see Lemma 1.1. Moreover, $\Gamma \neq\{0\}$ because each $\Gamma_{n}$ contains vectors with length one. It is a standard fact that we can choose a system of generators $\left\{w_{n}^{1}, w_{n}^{2}, w_{n}^{3}\right\}$ of $\Gamma_{n}$ for each $n$ such that there exists a subset $I \subset\{1,2,3\}$ and subsequences of $\left\{w_{n}^{i}\right\}$ that converge to $w^{i}$ when $i \in I$ and such that $\left\{w^{i}\right\}_{i \in I}$ are generators of $\Gamma$. The set $I$ is not empty because $\Gamma \neq\{0\}$. Now we are going to prove, reasoning by contradiction, that $\Gamma$ is a lattice in $\mathbb{R}^{3}$, i.e., that $\operatorname{rank}(\Gamma)=3$.

Assume that $I \neq\{1,2,3\}$. By suitably moving the surfaces $M_{n}$ we can suppose that all the surfaces $M_{n}$ contain the origin and that $\max _{p \in M_{n}}\left|\sigma_{n}\right|=\left|\sigma_{n}\right|(0)$. Note that, by Corollary $2.2, \operatorname{inj}\left(\mathbb{R}^{3} / \Gamma_{n}\right) \geq \frac{c\left(M_{n}\right)}{6} \geq \frac{1}{6 \max \left|\sigma_{n}\right|}$. Since $\left|\sigma_{n}\right|$ is uniformly bounded, a subsequence of $M_{n}$ converges by Corollary 2.8 in the $C^{k}$ topology, $k \geq 2$, to a properly embedded non flat minimal surface $M$, which is invariant by $\Gamma$. Moreover, as the index is clearly semicontinuous for the $C^{k}$ topology, we conclude that the index of $M / \Gamma$ is less than or equal to one and, as $M$ is non flat, we have that $\operatorname{Ind}(M / \Gamma)=1$.

Finally, as we are assuming that $\operatorname{rank}(\Gamma)=1,2$, we conclude from Proposition 3.1 that the total curvature of $M / \Gamma$ is $-4 \pi$. However, reasoning as in Assertion 3.1 , we prove that the total curvature of $M_{n} / \Gamma_{n}$ coincides with that of $M / \Gamma$, which is not possible because genus $\left(M_{n} / \Gamma_{n}\right) \geq 3$. This contradicton proves that $\Gamma$ is a lattice. In particular, $M / \Gamma$ is a compact minimal surface in the three torus $\mathbb{R}^{3} / \Gamma$ with the same topological type as $M_{n} / \Gamma_{n}$, and $M_{n} / \Gamma_{n}$ converges uniformly $C^{k}$, for $k \geq 2$, to $M / \Gamma$ by Theorem 2.8 .

Remark. As a consequence of the theorem, we obtain that the normalization on the injectivity radius of $\mathbb{R}^{3} / \Gamma_{n}$ can be replaced by other normalizations on geometrical invariants either of the tori or of the surfaces. In particular, we obtain the same compactness result by fixing either the area of the surfaces or the volume of the tori.

Now we identify homothetic flat three tori and consider the moduli space. Then we have

Corollary 3.3. The set of flat three tori that admit orientable embedded minimal surfaces with index one is a compact subset in moduli space. 
Proof. Suppose it is not true. Let $M_{n} / \Gamma_{n} \subset T_{n}^{3}=\mathbb{R}^{3} / \Gamma_{n}$ be a sequence of index one embedded minimal surfaces in flat three tori $T_{n}^{3}$ normalized in such a way that $\operatorname{inj}\left(T_{n}^{3}\right)=1$. By Theorem 3.2 a subsequence of $T_{n}^{3}$ converge to a torus $T^{3}$.

As the above moduli space is non compact, it follows that most three tori do not admit embedded orientable index one minimal surfaces. Recall that every flat three torus admits non orientable examples of minimal surfaces with index less than or equal to one, see $[\mathrm{HPR}]$ and $[\mathrm{M}]$.

\section{Stable Constant mean CURVature surfaces}

Let $M$ be an orientable stable constant mean curvature surface immersed into an orientable three dimensional Riemannian manifold $N$. A fundamental result for this kind of immersion is

Theorem 4.1. If $N$ is an orientable complete flat three manifold then either

(i) $M$ is totally geodesic, or

(ii) $M$ is compact and connected and genus $(M) \leq 5$.

Moreover, if genus $(M)=0$ or 1 then $M$ is an umbilical sphere or a flat torus, respectively, and if genus $(M)=4$ or 5 then $M$ is a minimal surface and $N$ is a flat three torus.

The proof of (i) and (ii) is outlined in [RR] and uses results of of Frensel [Fr], da Silveira [S], El Soufi and Ilias [EI], López and Ros [LR] and Yau [Y]. The classification of genus one stable surfaces is completed in $[R R]$ and the non existence of stable surfaces with genus 4 or 5 in complete orientable flat three manifolds different from $T^{3}$ is proved in $[\mathrm{R}]$.

When the ambient manifold is $\mathbb{R}^{3}$ it has been proved by Barbosa and do Carmo $[\mathrm{BdC}]$, Barbosa, do Carmo and Eschenburg [BdCE], El Soufi and Ilias [EI], and Heintze $[\mathrm{H}]$ that the only compact stable surface in $\mathbb{R}^{3}$ must be a totally umbilical sphere. For a general flat space form the only constant mean curvature immersion of a genus zero surface is the totally umbilical sphere, which is stable. If genus $(M)=1$, Ritoré and Ros [RR] have proved that $M$ must be a flat torus. In particular the only stable non totally geodesic surfaces with genus one are tubes around closed geodesics. It is also proved in $[\mathrm{RR}]$ that the following restriction on the signed curvature regions for a stable constant mean curvature non minimal surface holds: the region of negative Gauss curvature must be connected and each component of the region of positive Gauss curvature must contain an umbilic point at least. In higher genus no classification result is known. Some examples of stable triply periodic surfaces with genus three have been found by Ross, who has proved that the Schwarz minimal surfaces $\mathcal{P}$ and $\mathcal{D}$ are stable, see [Ro]. It follows from his results that each constant mean curvature surface close to the Schwarz $\mathcal{P}$ surface embedded in a three torus (not necessarily the cubic three torus) is stable.

Now we prove the main result of this section. Denote by $\mathcal{A}$ an affine conjugation class in $\mathcal{G}$ whose elements are not cyclic subgroups. Recall that there are only a finite number of such classes.

Theorem 4.2 (Compactness of the space of stable surfaces). Let $\left\{M_{n}\right\}_{n \in \mathbb{N}}$ be a sequence of connected embedded constant mean curvature surfaces in $\mathbb{R}^{3}$ and $\left\{G_{n}\right\}_{n \in \mathbb{N}}$ a sequence in $\mathcal{A}$ normalized in such a way that $\operatorname{inj}\left(\mathbb{R}^{3} / G_{n}\right)=1$. Suppose that $M_{n}$ is invariant by $G_{n}$ and that the surfaces $M_{n} / G_{n}$ are compact, orientable and stable in $\mathbb{R}^{3} / G_{n}$ with fixed genus greater than one. Then 
(i) We can extract a subsequence of $G_{n}$, denoted in the same way, that converges to a subgroup $G \in \mathcal{A}$, i.e., $\mathbb{R}^{3} / G_{n}$ and $\mathbb{R}^{3} / G$ are affinely diffeomorphic.

(ii) We can extract a subsequence of $M_{n}$, denoted in the same way, converging, up to translations in $\mathbb{R}^{3}$, in the $C^{k}$ topology to a connected properly weakly embedded constant mean curvature $G$-invariant surface $M$, with multiplicity one, in $\mathbb{R}^{3}$ such that $M / G$ is a compact stable surface diffeomorphic to $M_{n} / G_{n}$.

Proof. Let us see first that there exists a constant $C>0$ such that $\left|\sigma_{n}\right|<C$ for all $n \in \mathbb{N}$. If not, there would exist a sequence $p_{n} \in M_{n}$ such that $\lambda_{n}=$ $\max _{p \in M_{n}}\left|\sigma_{n}\right|=\left|\sigma_{n}\right|\left(p_{n}\right) \rightarrow \infty$. By moving the points $p_{n}$ to the origin, expanding the surfaces $M_{n}$ by a factor $\lambda_{n}$ and passing to a subsequence if neccesary, we obtain a sequence of properly embedded constant mean curvature surfaces $\widetilde{M}_{n}$ converging in the $C^{k}$ topology to a non totally geodesic properly weakly embedded constant mean curvature surface $\widetilde{M}$ by Theorem 2.8. The surface $\widetilde{M}$ is orientable: in fact, if $\widetilde{M}$ were non orientable, its multiplicity should be two (otherwise, from Theorem $2.8 \widetilde{M}$ would have multiplicity one and in this case the orientability of $\widetilde{M}_{n} / \lambda_{n} G_{n}$ implies that of $\widetilde{M}$ ) and, again by Theorem $2.8, \widetilde{M}$ would be a properly embedded minimal surface in $\mathbb{R}^{3}$, which is impossible. As $\operatorname{inj}\left(\mathbb{R}^{3} / G_{n}\right)=1$, it follows that the only accumulation point of the sequence of subgroups $\left\{\lambda_{n} G_{n}\right\}_{n \in \mathbb{N}}$ in the isometry group Iso $\left(\mathbb{R}^{3}\right)$ is the identity map. Then compact regions in $\widetilde{M}$ can be uniformly approximated by regions in $\widetilde{M}_{n} / \lambda_{n} G_{n}$ and, as the stability is clearly preserved by the $C^{k}$ convergence, $k \geq 2$, we conclude that $\widetilde{M}$ is a stable surface in $\mathbb{R}^{3}$ and, from Theorem 4.1, that $\widetilde{M}$ is a round sphere. By applying Lemma 2.7 to the surfaces $\widetilde{M}_{n}$ and to the groups $\lambda_{n} G_{n}$ we conclude that the sphere $\widetilde{M}$ is non compact. This contradiction proves that the sequence $\left|\sigma_{n}\right|$ is uniformly bounded.

From the normalization $\operatorname{inj}\left(\mathbb{R}^{3} / G_{n}\right)=1$ it follows, passing to a subsequence, that the subgroups $\left\{G_{n}\right\}_{n \in \mathbb{N}}$ converge to a subgroup $G \in \mathcal{G}$. By Corollary 2.2, we obtain $1=\operatorname{inj}\left(\mathbb{R}^{3} / G_{n}\right) \geq \frac{c(M)}{6} \geq \frac{1}{\max \left|\sigma_{n}\right|}$. So, suitably moving the surfaces $M_{n}$ in $\mathbb{R}^{3}$, we can suppose that a subsequence of $\left\{M_{n}\right\}_{n \in \mathbb{N}}$ converges to a non totally geodesic properly weakly embedded constant mean curvature $G$-invariant surface $M$. We claim that $M / G$ is compact: in fact, if $M / G$ is orientable, then it is stable, and so it is compact by Theorem 4.1. If $M / G$ is non orientable, then its multiplicity must be two (otherwise, from Theorem $2.8 \mathrm{M} / G$ would have multiplicity one and, as before, the orientability of $M_{n} / G_{n}$ implies that of $M / G$ ). Moreover, the orientation double cover is stable and hence compact, and it follows that $M / G$ is compact. This proves our claim. Finally, we conclude from (ii) in Theorem 2.8 that $G \in \mathcal{A}$, that $M_{n} / G_{n}$ and $M / G$ are diffeomorphic and that $M_{n} / G_{n} \rightarrow M / G$ with multiplicity one.

Remark. There is no compactness in a strong sense for the space of genus one stable embedded surfaces. In fact, if we consider the quotient $\mathbb{R}^{3} / T_{v}$, where $T_{v}$ is the subgroup generated by a non trivial translation, the tubes around a closed geodesic in $\mathbb{R}^{3} / T_{v}$ are stable embedded constant mean curvature surfaces for large enough radius. But these tubes, suitably translated, converge to a totally geodesic cylinder. So the topology is not preserved in the limit.

We have needed the connectedness of the surfaces $M_{n}$ in the above theorem to prove the compactness of the ambient manifolds $\mathbb{R}^{3} / G_{n}$ and to ensure that the limit surface is of multiplicity one. If we omit these hypothesis and we take these 
three manifolds in a compact subset, by using the same arguments, we get a similar result. In particular, fixing the ambient manifold, we obtain

Theorem 4.3. Let $\left\{M_{n}\right\}_{n \in \mathbb{N}}$ be a sequence of embedded constant mean curvature surfaces in $\mathbb{R}^{3}$ and $G \in \mathcal{G}$ such that the surfaces $M_{n}$ are invariant by $G$. Suppose that $M_{n} / G$ are compact, orientable and stable surfaces in $\mathbb{R}^{3} / G$ with genus $\left(M_{n} / G\right) \geq 2$.

Then we can extract a subsequence of $M_{n}$, denoted in the same way, that converges to a properly weakly embedded constant mean curvature surface $M$ in $\mathbb{R}^{3}$ and is such that either

(i) $M / G$ is an orientable stable surface diffeomorphic to $M_{n} / G$ for $n$ large enough, or

(ii) $M / G$ is a compact non orientable minimal surface with multiplicity two whose orientable double cover is stable and diffeomorphic to $M_{n} / G$ for $n$ large enough.

The hypothesis in Theorem 4.2 on the connectedness of the surfaces $M_{n}$, which is equivalent to the surjectivity of the induced map from the inclusion $M_{n} / G_{n} \subset$ $\mathbb{R}^{3} / G_{n}$ between the fundamental group, is always true if the surfaces are non flat and minimal. The following lemma gives us another situation for which this property holds

Lemma 4.4. If $M \subset \mathbb{R}^{3}$ is a properly embedded surface with constant mean curvature invariant by a rank two subgroup $\Gamma$ of translations of $\mathbb{R}^{3}$ and such that $M / \Gamma$ is a compact and connected surface with genus greater than one, then $M$ is connected.

Proof. Let $V$ be the plane determined by $\Gamma$ and $T$ the torus $V / \Gamma$. By Alexandrov's reflection principle we can suppose that the surface $M / \Gamma$ is symmetric with respect to the totally geodesic torus $T \times\{0\} \subset T \times \mathbb{R}$ and is the union of two graphs over $T \times\{0\}$. Let $\psi: M / \Gamma \rightarrow T \times \mathbb{R}$ be the inclusion map and $\Gamma^{\prime}=\psi_{*}\left(\pi_{1}(M / \Gamma)\right)$. Then $\operatorname{rank}\left(\Gamma^{\prime}\right)$ must be two: if not, $M / \Gamma$ could be lifted to $\mathbb{R}^{3} / \Gamma^{\prime}$, and so genus $(M / \Gamma)=$ 0 or 1 . Let $T^{\prime}=V / \Gamma^{\prime}$. The group $\Gamma / \Gamma^{\prime}$ is finite and we have a non trivial finite Riemannian covering $T^{\prime} \times \mathbb{R} \rightarrow T \times \mathbb{R}$ such that the inverse image of $M / \Gamma$ is a finite collection of connected disjoint surfaces $M_{1}, \ldots, M_{k}$ congruent by translations of $\Gamma$ and each of them congruent to $M / \Gamma$. If $\Pi: T^{\prime} \times \mathbb{R} \rightarrow T^{\prime} \times\{0\}$ is the projection on $T^{\prime}$, we have that the subsets $\Pi\left(M_{i}\right)$ are disjoint. Moreover, the embeddings $\psi_{i}: M_{i} \rightarrow T^{\prime} \times \mathbb{R}$ induce, between the fundamental groups, a surjective map $\psi_{i *}: \pi_{1}\left(M_{i}\right) \rightarrow \pi_{1}\left(T^{\prime}\right)$.

The connectedness of $M$ is equivalent to the equality $\Gamma^{\prime}=\Gamma$. We suppose, reasoning by contradiction, that $\Gamma^{\prime} \neq \Gamma$ and so, that $k \geq 2$. Since the fundamental group of $T^{\prime} \times \mathbb{R}$ is abelian, and $\psi_{i_{*}}$ is surjective, we deduce that the morphism induced by $\psi_{i}$ between the first homology groups is also surjective for all $i \in\{1, \ldots, k\}$ and, since $\Pi$ induces a isomorphism in homology, we have that $\Pi \circ \psi_{i}$ induces a surjective mapping between the homology groups $H_{1}\left(M_{i}, \mathbb{Z}\right)$ and $H_{1}\left(T^{\prime}, \mathbb{Z}\right)$. Then we can take $\alpha, \beta$ cycles in $M_{1}$ and $M_{2}$, respectively, such that $\Pi\left(\psi_{1}(\alpha)\right)$ and $\Pi\left(\psi_{2}(\beta)\right)$ are representants of a basis of $H_{1}\left(T^{\prime}, \mathbb{Z}\right)$. But the last two cycles are disjoint, which is impossible, because the intersection number of a basis in the first homology group of a two torus must be \pm 1 . This contradiction shows that $M$ is connected.

We identify homothetical non compact complete orientable flat three dimensional manifolds and consider the moduli space. We obtain 
Corollary 4.5. The set of complete non compact orientable flat three dimensional manifolds that admit compact orientable embedded stable surfaces with genus greater than one is a compact subset in moduli space.

Proof. Let $M_{n} / G_{n} \subset \mathbb{R}^{3} / G_{n}$ be a sequence satisfying the conditions of the corollary. Since genus $\left(M_{n} / G_{n}\right) \geq 2$ we have that $\operatorname{rank}\left(\Gamma\left(G_{n}\right)\right) \geq 2$ and, as $\mathbb{R}^{3} / G_{n}$ is non compact, we conclude that $\operatorname{rank}\left(\Gamma\left(G_{n}\right)\right)=2$. So we have two different affine diffeomorphism classes in the sequence $\mathbb{R}^{3} / G_{n}$ and we can suppose, passing to a subsequence, that all the manifolds $\mathbb{R}^{3} / G_{n}$ are of the same type.

If $\mathbb{R}^{3} / G_{n}=T_{n}^{2} \times \mathbb{R}$ for all $n \in \mathbb{N}$, where $T_{n}^{2}$ is a flat two dimensional torus, then Lemma 4.4 allows us to use Theorem 4.2 to complete the proof. If all the manifolds $\mathbb{R}^{3} / G_{n}$ lie in the class $\mathcal{K}$ and, for a subsequence, the morphisms induced between the fundamental groups by the inclusions are surjective, then we conclude again by Theorem 4.2 the existence of the limit.

If $\mathbb{R}^{3} / G_{n} \in \mathcal{K}$, but the morphisms between the fundamental groups are not surjective, we consider twofold coverings $T_{n}^{2} \times \mathbb{R} \rightarrow \mathbb{R}^{3} / G_{n}$. By Lemma 4.4 the pullback of $M_{n} / G_{n}$ in $T_{n}^{2} \times \mathbb{R}$ is not connected, and so $M_{n} / G_{n}$ lifts to $T_{n}^{2} \times \mathbb{R}$. Hence we can extract a convergent subsequence of $T_{n}^{2} \times \mathbb{R}$ and so of $\mathbb{R}^{3} / G_{n}$.

Remark. As the above moduli space is non compact it follows from Corollary 4.5 that for most non compact orientable flat three manifolds the only compact orientable stable surfaces have genus less than or equal to one and, by Theorem 4.1, they are either umbilical spheres or flat tori. In particular, the isoperimetric domains in most of these manifolds are necessarily geodesic balls, tubular neighborhoods of closed geodesics, and tubular neighborhoods of totally geodesic tori.

Remark. The result about existence of stable constant mean curvature surfaces by Ross [Ro] suggests that it will be difficult to give an explicit description of all stable constant mean curvature and index one minimal surfaces. The compactness theorems we have proved show that the spaces of stable constant mean curvature surfaces and index one minimal surfaces are small in some sense. For instance, it can be deduced from our results that the number of homotopy classes of stable constant mean curvature and index one minimal surfaces in an affine diffeomorphism class of complete flat three manifolds is finite. Up to now, the existence of genus four or five index one minimal surfaces in flat three tori and the existence of a stable surface with genus two is not known.

We should also mention that there are non-compact families of non-orientable minimal surfaces with index at most one in flat three tori, see $[\mathrm{HPR}]$ and $[\mathrm{M}]$.

The methods we have used fail in proving the compactness of the space of index one constant mean curvature surfaces. From the behaviour of the uniparametric family of doubly periodic constant mean curvature square grids derived from Lawson's examples, [Kar], it seems likely that this space is non compact in general.

\section{REFERENCES}

[A] A.D. Alexandrov, Uniqueness theorems for surfaces in the large, I, Vestnik Leningrad Univ. Math. 11 (1956), no. 19, 5-17; English transl., Amer. Math. Soc. Transl. (2) 21 (1962), 341-353. MR 19:167; MR 27:698a

$[\mathrm{BdC}]$ J.L. Barbosa, M. do Carmo, Stability of hypersurfaces with constant mean curvature, Math. Z. 185 (1984), 339-353. MR 85k:58021c 
[BdCE] J.L. Barbosa, M. do Carmo, J. Eschenburg, Stability of hypersurfaces with constant mean curvature in Riemannian manifolds, Math. Z. 197 (1988), 123-138. MR 88m:53109

[dCP] M. do Carmo, C.K. Peng, Stable complete minimal surfaces in $\mathbb{R}^{3}$ are planes, Bull. Amer. Math. Soc. (N.S.) 1 (1979), 903-906. MR 80j:53012

[EI] A. El Soufi, S. Ilias, Majoration de la seconde valeur propre d'un opérateur de Schrödinger sur une variété compacte et applications, J. Funct. Anal. 103 (1992), 294316. MR 93g:58150

[FC] D. Fischer-Colbrie, On complete minimal surfaces with finite Morse index in threemanifolds, Invent. Math. 82 (1985), 121-132. MR 87b:53090

[FCS] D. Fischer-Colbrie, R. Schoen, The structure of complete stable minimal surfaces in 3-manifolds of non-negative scalar curvature, Comm. Pure Appl. Math. 33 (1980), 199-211. MR 81i:53044

[Fr] K. Frensel, Stable complete surfaces with constant mean curvature, An. Acad. Brasil. Ciênc. 60 (1988), 115-117. MR 90c:53019

[GH] P. Griffiths, J. Harris, Principles of Algebraic Geometry, Pure and Applied Math., Wiley-Interscience, New York, 1978. MR 80b:14001

[HPR] J. Hass, J.T. Pitts, J.H. Rubinstein, Existence of unstable minimal surfaces in manifolds with homology and applications to triply periodic minimal surfaces, Proceedings of Symposia in Pure Mathematics, vol. 54, part 1, Amer. Math. Soc., 1993, pp. 147-162. MR 94j:53007

[H] E. Heintze, Extrinsic upper bounds for $\lambda_{1}$, Math. Ann. 280 (1988), 389-402. MR 89f:53091

[HM] D. Hoffman, W.H. Meeks, Limits of minimal surfaces and Scherk's second surface, Univ. Massachusetts.

[HM1] D. Hoffman, W.H. Meeks, The strong halfspace theorem for minimal surfaces, Invent. Math. 101 (1990), 373-377. MR 92e:53010

[Kar] H. Karcher, The triply periodic minimal surfaces of Alan Schoen and their constant mean curvature companions, Manuscripta Math. 64 (1989), 291-357. MR 90g:53010

[KKS] N.J. Korevaar, R. Kusner, B. Solomon, The structure of complete embedded surfaces with constant mean curvature, J. Differential Geom. 30 (1989), 465-503. MR 90g:53011

[LR] F.J. López, A. Ros, Complete minimal surfaces with index one and stable constant mean curvature surfaces, Comment. Math. Helv. 64 (1989), 34-43. MR 90b:53006

[M] W.H. Meeks, The theory of triply periodic minimal surfaces, Indiana U. Math. J. 39 (1990), 877-936. MR 92e:53012

[MR1] W.H. Meeks, H. Rosenberg, The global theory of doubly periodic minimal surfaces, Invent. Math. 97 (1989), 351-379. MR 90m:53017

[MR2] W.H. Meeks, H. Rosenberg, The geometry of periodic minimal surfaces, Comment. Math. Helv. 68 (1993), 538-579. MR 95a:53011

[MoR] S. Montiel, A. Ros, Schrödinger operators associated to a holomorphic map, Proceedings Conference on Global Differential Geometry and Global Analysis, Lecture Notes in Mathematics 1481, Berlin, 1991, 147-174. MR 93k:58053

[P] J.T. Pitts, Existence and regularity of minimal surfaces on Riemannian manifolds, Mathematical Notes 27, Princeton University Press, Princeton, 1981. MR 83e:49079

[PR] J.T. Pitts, J.H. Rubinstein, Equivariant minimax and minimal surfaces in geometric three-manifolds, Bull. Amer. Math. Soc. (N.S.) 19 (1988), 303-309. MR 90a:53014

[R] M. Ritoré, Complete orientable index one minimal surfaces embedded in complete orientable flat three manifolds, preprint, Univ. Granada, 1994.

[RR] M. Ritoré, A. Ros, Stable constant mean curvature tori and the isoperimetric problem in three space forms, Comment. Math. Helv. 67 (1992), 293-305. MR 93a:53055

[Ro] M. Ross, Schwarz's $\mathcal{P}$ and $\mathcal{D}$ surfaces are stable, Differential Geom. Appl. 2 (1992), 179-195. MR 94j:53010

[Sc] R. Schoen, Uniqueness, symmetry and embeddedness of minimal surfaces, J. Differential Geom. 18 (1983), 791-809. MR 85f:53011

[S] A. da Silveira, Stability of complete noncompact surfaces with constant mean curvature, Math. Ann. 277 (1987), 629-638. MR 88h:53053

[W] B. White, Curvature estimates and compactness theorems in 3-manifolds for surfaces that are stationary for parametric elliptic functionals, Invent. Math. 88 (1987), 243-256. MR 88g:58037 
[Wo] J.A. Wolf, Spaces of constant curvature, 1st ed., Publish or Perish, Inc., 1984.

[Y] S.T. Yau, Nonlinear analysis in geometry, L'Enseignement Math. 33 (1987), $109-158$. MR 88g:58003

Departamento de Geometría y Topología, Universidad de Granada, E-18071, Granada, SPAIN

E-mail address: mritore@ugr.es

Departamento de Geometría y Topología, Universidad de Granada, E-18071, Granada, SPAIN

E-mail address: aros@ugr.es 